\title{
Robust Learning StabiLity With Operational Monetary Policy Rules
}

\author{
George W. Evans \\ University of Oregon and University of St Andrews \\ Seppo Honkapohja \\ Bank of Finland and University of Cambridge
}

The recent literature examines the conduct of monetary policy in terms of interest rate rules from the viewpoint of imperfect knowledge and learning by economic agents. The stability of the rational expectations equilibrium is taken as a key desideratum for good monetary policy design. ${ }^{1}$ Most of this literature postulates that agents use least squares or related learning algorithms to carry out real-time estimations of the parameters of their forecast functions as new data become available. Moreover, it is usually assumed that the learning algorithms have a decreasing gain; in the most common case, the gain is the inverse of the sample size so that all data points have equal weights. Use of such a decreasing-gain algorithm makes it possible for learning to converge exactly to the rational expectations equilibrium in environments without structural change. Convergence requires that the equilibrium satisfies a stability condition, known as E-stability.

Decreasing-gain algorithms do not perform well, however, when occasional unobservable structural changes take place. So-called constant-gain algorithms are a natural alternative for estimating parameters in a way that is alert to possible structural changes. If agents use a constant-gain algorithm, then parameter estimates of the forecast functions do not fully converge to the rational expectations

We thank our discussant Claes Berg, Carl E. Walsh, and the conference participants for valuable comments. Support from National Science Foundation Grant No. SES0617859 and ESRC grant RES-000-23-1152 is gratefully acknowledged.

1. For surveys, see Evans and Honkapohja (2003a), Bullard (2006), and Evans and Honkapohja (in this volume).

Monetary Policy under Uncertainty and Learning, edited by Klaus Schmidt-Hebbel and Carl E. Walsh, Santiago, Chile. C 2009 Central Bank of Chile. 
equilibrium values. Instead, they remain random, even asymptotically. For small values of the gain parameter, the estimates mostly remain in a small neighborhood of the rational expectations equilibrium, provided that the equilibrium is E-stable. ${ }^{2}$ Constant-gain algorithms have recently been employed in empirical work, such as Milani (2005, 2007a), Orphanides and Williams (2005a, 2005b), and Branch and Evans (2006).

The connection between convergence of constant-gain learning and E-stability noted above is a limiting result for sufficiently small gain parameters. For finite values of the gain parameter, the stability condition for constant-gain learning is more stringent than E-stability. In this paper we examine the stability implications of various interest rate rules when agents use constant-gain learning rules with plausible positive values of the gain. We say that an interest rate rule yields robust learning stability of the economy if stability under constant-gain learning obtains for all values of the gain parameter in the range suggested by the empirical literature. ${ }^{3}$ We focus on interest rate rules that are operational in the sense discussed by McCallum (1999), who holds that monetary policy cannot be conditioned on current values of endogenous aggregate variables. The rules we consider therefore assume that policy responds to expectations of contemporaneous (or future) values of inflation and output, but not on their actual values in the current period.

We consider robust learning stability for a variety of operational interest rate rules that have been suggested in the recent literature. These include Taylor rules and optimal reaction functions under discretion and commitment when central bank policy aims for interest rate stabilization in addition to the usual motives for flexible inflation targeting. The reaction function may be expectations-based in the spirit of Evans and Honkapohja (2003b, 2006) or of the Taylor-type form suggested by Duffy and Xiao (2007). We also analyze two interest

2. See Evans and Honkapohja (2001, chaps. 3 and 7) for the basic theoretical results on constant-gain learning. See also Evans, Honkapohja, and Williams (forthcoming) for references on recent papers on constant-gain learning. The possibility of divergence resulting from constant gain learning was noted in Slobodyan, Bogomolov, and Kolyuzhnov (2006).

3. Numerous concepts of robustness are relevant to policymaking, reflecting, for example, uncertainty about the structure of the economy and a desire by both private agents and policymakers to guard against the risk of large losses. We do not mean to downplay the importance of such factors, but we abstract from them here to focus on the importance of setting policy in such a way as to ensure stability in the face of constant-gain learning. 
rate rules that approximate optimal policy under commitment, as suggested by Svensson and Woodford (2005) and McCallum and Nelson (2004). Our results show that expectations-based rules deliver robust learning stability, whereas the proposed alternatives often become unstable under learning even at quite small values of the constant-gain parameter.

\section{Constant-Gain Steady-State Learning}

In this paper we employ multivariate linear models. In this simplest case, in which the shocks are white noise and there are no lagged endogenous variables, the rational expectations equilibrium takes the form of a stochastic steady state. We now briefly review the basics of steady-state learning in linear models and then apply the results to Taylor rules. ${ }^{4}$

\subsection{Theoretical Results}

The steady state can be computed by postulating that agents' beliefs, called the perceived law of motion (PLM), take the form

$\mathbf{y}_{t}=\mathbf{a}+\mathbf{e}_{t}$

for a vector $\mathbf{y}_{t}$, where $\mathbf{e}_{t} \sim$ i.i.d. $\left(0, \sigma^{2}\right)$. Using the model, one then computes the actual law of motion (ALM), which describes the temporary equilibrium in the current period, given the PLM. We write the ALM using a linear operator $\mathbf{T}$ as

$\mathbf{y}_{t}=\mathbf{\alpha}+\mathbf{T a}+\mathbf{e}_{t}$

where the matrix $\mathbf{T}$ depends on the structural parameters of the model. Examples of the $\mathbf{T}$ map are provided below. A rational expectations equilibrium is a fixed point, $\overline{\mathbf{a}}$, of the $\mathbf{T}$ map, that is,

$\overline{\mathbf{a}}=\boldsymbol{\alpha}+\mathbf{T} \overline{\mathbf{a}}$.

We assume that $\mathbf{I}-\mathbf{T}$ is nonsingular, so that there is a unique solution $\overline{\mathbf{a}}=(\mathbf{I}-\mathbf{T})^{-1} \boldsymbol{\alpha}$. For convenience, and without loss of generality, we

4. See Evans and Honkapohja (2001, chaps. 8 and 10) for a detailed discussion of adaptive learning in linear models. 
now assume that the model has been written in deviation-from-themean form, so that $\boldsymbol{\alpha}=\mathbf{0}$. Thus the rational expectations equilibrium corresponds to $\overline{\mathbf{a}}=\mathbf{0}$ in our analysis. Under learning, agents attempt to learn the value of $\overline{\mathbf{a}}$, and hence in deviation-from-the-mean form we are examining whether agents' estimates of the mean converge to $\mathbf{a}=\mathbf{0}$.

Steady-state learning under decreasing gain is given by the recursive algorithm,

$\mathbf{a}_{t}=\mathbf{a}_{t-1}+\gamma_{t}\left(\mathbf{y}_{t}-\mathbf{a}_{t-1}\right)$,

where the gain $\gamma_{t}$ is a sequence of small decreasing numbers, such as $\gamma_{t}=1 / t$. Assuming that $\mathbf{y}_{t}=\mathbf{T} \mathbf{a}_{t-1}+\mathbf{e}_{t}$, that is, that expectations are formed using the estimate $\mathbf{a}_{t-1}$ based on data through time $t-1$, the convergence condition of algorithm (1) is given by the conditions for local asymptotic stability of $\overline{\mathbf{a}}$ under an associated differential equation:

$$
\frac{d \mathbf{a}}{d \tau}=\mathbf{T a}-\mathbf{a},
$$

which is known as the E-stability differential equation. Here $\tau$ denotes notional or virtual time. The E-stability condition holds if and only if all eigenvalues of the matrix $\mathbf{T}$ have real parts less than one. ${ }^{5}$

Under constant-gain learning, the estimate $\mathbf{a}_{t}$ of $\mathbf{a}$ is updated according to

$\mathbf{a}_{t}=\mathbf{a}_{t-1}+\gamma\left(\mathbf{y}_{t}-\mathbf{a}_{t-1}\right)$,

where $0<\gamma \leq 1$ is the constant-gain parameter. The only difference between equation (2) and equation (1) is the constancy of the gain sequence. We now have

$\mathbf{a}_{t}=\mathbf{a}_{t-1}+\gamma\left(\mathbf{T} \mathbf{a}_{t-1}+\mathbf{e}_{t}-\mathbf{a}_{t-1}\right)$,

or

$\mathbf{a}_{t}=[\gamma \mathbf{T}+(1-\gamma) \mathbf{I}] \mathbf{a}_{t-1}+\gamma \mathbf{e}_{t}$

5. Throughout, we rule out boundary cases in which the real part of some eigenvalue of the $\mathbf{T}$ map is one. 
This converges to a stationary stochastic process around the rational expectations equilibrium value (in deviation-from-the-mean form) provided all roots of the matrix $\gamma \mathbf{T}+(1-\gamma) \mathbf{I}$ lie inside the unit circle.

Stability under constant-gain learning depends on the value of $\gamma$, and we have the following result.

Proposition 1. For a given $0<\gamma \leq 1$, the stability condition is that the eigenvalues of $\mathbf{T}$ lie inside a circle of radius $1 / \gamma$ and origin at $(1-1 / \gamma, 0)$. This condition is therefore stricter for larger values of $\gamma$.

Proof. The stability condition is that the roots of $\gamma\left[\mathbf{T}+\gamma^{-1}(1-\gamma) \mathbf{I}\right]$ lie inside the unit circle centered at the origin. Equivalently, the roots of $\left[\mathbf{T}+\gamma^{-1}(1-\gamma) \mathbf{I}\right]$ must lie inside a circle of radius $1 / \gamma$ centered at the origin. Since the roots of $\mathbf{T}+\gamma^{-1}(1-\gamma) \mathbf{I}$ are the same as the roots of $\mathbf{T}$ plus $\gamma^{-1}(1-\gamma)$, this is equivalent to the condition given.

The right edge of the circle is at $(1,0)$ in the complex plane, and as $\gamma \rightarrow 0$ we obtain the standard (decreasing-gain) E-stability condition that the real parts of all roots of $\mathbf{T}$ are less than one. Looking at the other extreme, $\gamma=1$, gives the following corollary of proposition 1 :

Proposition 2. We have stability for all $0<\gamma \leq 1$ if and only if all eigenvalues of $\mathbf{T}$ lie inside the unit circle.

Stability for all constant gains, $0<\gamma \leq 1$, is equivalent to a condition known as iterative E-stability, sometimes called IE-stability. Iterative E-stability is said to hold when $\mathbf{T}^{j} \rightarrow \mathbf{0}$ as $j \rightarrow \infty$. $^{6}$

When the stability condition holds, the parameter $\mathbf{a}_{t}$ converges to a stationary stochastic process that we can fully describe. This, in turn, induces a stationary stochastic process for $\mathbf{y}_{t}=\mathbf{T a} \mathbf{a}_{t-1}+\mathbf{e}_{t}$.

\subsection{Application to Taylor Rules}

Consider the standard forward-looking New-Keynesian model,

$$
\begin{aligned}
& x_{t}=-\varphi\left(i_{t}-\pi_{t+1}^{e}\right)+x_{t+1}^{e}+g_{t} ; \\
& \pi_{t}=\lambda x_{t}+\beta \pi_{t+1}^{e}+u_{t} .
\end{aligned}
$$

For convenience we assume that $\left(g_{t}, u_{t}\right)^{\prime}$ are independent and identically distributed (i.i.d.), so that the preceding technical results

6. In many models, iterative E-stability is known to be a necessary condition for the stability of eductive learning; see, for example, Evans and Guesnerie (1993). 
can be applied. Later we consider cases with first-order autoregressive, or AR(1), shocks. We use $x_{t+1}^{e}$ and $\pi_{t+1}^{e}$ to denote expectations of $\pi_{t+1}$ and $x_{t+1}$. Below we specify the information sets available to agents when they are forming expectations, and throughout the paper we explore the implications of alternative assumptions.

Bullard and Mitra (2002) consider Taylor rules of various forms, including the contemporaneous data rule,

$i_{t}=\chi_{\pi} \pi_{t}+\chi_{x} x_{t}$,

and the "contemporaneous expectations" rule,

$i_{t}=\chi_{\pi} \pi_{t}^{e}+\chi_{x} x_{t}^{e}$.

In this section, our analysis of the contemporaneous expectations rule follows Bullard and Mitra (2002) in assuming that all expectations are based on information at time $t-1$, that is, $\pi_{t}^{e}=\hat{E}_{t-1} \pi_{t}, x_{t}^{e}=\hat{E}_{t-1} x_{t}$, $\pi_{t+1}^{e}=\hat{E}_{t-1} \pi_{t+1}$, and $x_{t+1}^{e}=\hat{E}_{t-1} x_{t+1}$. Since we have i.i.d. shocks, forecasts are based purely on the estimated intercept.

Bullard and Mitra (2002) show that the determinacy and Estability conditions are the same and are identical for both interest rate rules. They are given by

$\lambda\left(\chi_{\pi}-1\right)+(1-\beta) \chi_{x}>0$.

Bullard and Mitra consider this finding important because of McCallum's (1999) argument that interest rate rules cannot plausibly be conditioned on contemporaneous observations of endogenous aggregate variables like inflation and output, whereas they could plausibly be conditioned on central bank forecasts or "nowcasts" $\hat{E}_{t-1} \pi_{t}, \hat{E}_{t-1} x_{t}$.

We reconsider this issue from the vantage point of constant-gain learning. For the interest rate rule (6), the model takes the form

$$
\mathbf{y}_{t}=\mathbf{M}_{0} \mathbf{y}_{t}^{e}+\mathbf{M}_{1} \mathbf{y}_{t+1}^{e}+\mathbf{P} \mathbf{v}_{t}
$$

where $\mathbf{y}_{t}^{\prime}=\left(x_{t}, \pi_{t}\right)$ and $\mathbf{v}_{t}^{\prime}=\left(g_{t}, u_{t}\right)$ and where

$$
\mathbf{M}_{0}=\left(\begin{array}{cc}
-\chi_{x} \varphi & -\chi_{\pi} \varphi \\
-\chi_{x} \varphi \lambda & -\chi_{\pi} \varphi \lambda
\end{array}\right) \text { and } \mathbf{M}_{1}=\left(\begin{array}{cc}
1 & \varphi \\
\lambda & \beta+\varphi \lambda
\end{array}\right),
$$


and

$\mathbf{P}=\left(\begin{array}{cc}1 & 0 \\ \lambda & 1\end{array}\right)$

Since our shocks are i.i.d., the PLM is simply $\mathbf{y}_{t}=\mathbf{a}+\mathbf{e}_{t}$, and the corresponding ALM is $\mathbf{y}_{t}=\left(\mathbf{M}_{0}+\mathbf{M}_{1}\right) \mathbf{a}+\mathbf{e}_{t}$, where $\mathbf{e}_{t}=\mathbf{P} \mathbf{v}_{\mathrm{t}}$. The usual E-stability condition is that the eigenvalues of $\mathbf{M}_{0}+\mathbf{M}_{1}$ have real parts less than one, which leads to condition (7). According to proposition 2 , for convergence of constant-gain learning for all gains $0<\gamma \leq 1$, both eigenvalues of $\mathbf{M}_{0}+\mathbf{M}_{1}$ must lie inside the unit circle.

We investigate the stability of constant-gain learning numerically, using the Woodford calibration of $\varphi^{-1}=0.157, \lambda=0.024, \beta=0.99$. Setting $\chi_{\pi}=1.5$, eigenvalues with real parts less than -1 arise for $\chi_{x}>0.31$ and eigenvalues with real parts less than -9 arise for $\chi_{x}>1.57$. This implies that when $\chi_{\pi}=1.5$ and $\chi_{x}>1.57$, the equilibrium is unstable under learning for constant gains $\gamma \geq 0.10$. This is perhaps not a significant practical concern since Taylor's recommended parameters are $\chi_{\pi}=1.5$ and (based on the quarterly calibration of Woodford) $\chi_{x}=(0.5) / 4=0.125$. However, it does show a previously unrecognized danger that arises under constant-gain learning if the Taylor rule has too strong a response to $\hat{E}_{t-1} x_{t}$, and this finding foreshadows instability problems that arise in more sophisticated rules discussed below.

Finally, the potential for instability under constant-gain learning arises specifically because of the need to use forecasts $\hat{E}_{t-1} \mathbf{y}_{t}$. For the current-data Taylor rule (5), it can be shown that condition (7) guarantees stability under learning for all constant gains $0<\gamma \leq 1$. $^{7}$

\section{Optimal Discretionary Monetary Policy}

We now consider optimal policy under constant-gain learning, starting with optimal discretionary policy. We focus on homogeneous learning by private agents and the policymaker. We initially restrict attention to the case of i.i.d. exogenous shocks, so that steady-state

7. The model now takes the form $\mathbf{y}_{t}=\mathbf{M}_{1} \hat{E}_{t} \mathbf{y}_{t+1}+\mathbf{P v}_{t}$, and the required condition is the same as the determinacy condition. 
learning is appropriate. We also analyze the more general case, in which the observable shocks follow AR(1) processes.

Consider the loss function

$E_{0} \sum_{t=0}^{\infty}\left[\left(\pi_{t}-\pi^{*}\right)^{2}+\alpha_{x}\left(x_{t}-x^{*}\right)^{2}+\alpha_{i}\left(i_{t}-i^{*}\right)^{2}\right]$

where $\pi^{*}, x^{*}$, and $i^{*}$ represent target values. For simplicity, we set $\pi^{*}=x^{*}=0$. The weights $\alpha_{x}, \alpha_{i}>0$ represent relative weights given by policymakers to squared deviations of $x_{t}$ and $i_{t}$ from their targets, compared with squared deviations of $\pi_{t}$ from its target.

The first-order condition for discretionary optimal policy is

$\lambda \pi_{t}+\alpha_{x} x_{t}-\alpha_{i} \varphi^{-1}\left(i_{t}-i^{*}\right)=0$.

We first consider a Taylor-Type Rule proposed by Duffy and Xiao (2007) and then discuss the expectations-based rule recommended by Evans and Honkapohja (2003b).

\subsection{Taylor-Type Optimal Rules}

Duffy and Xiao (2007) propose using the equation (11) directly to obtain a Taylor-Type Rule that implements optimal discretionary policy. Solving the first-order condition for $i_{t}$ yields the rule

$$
i_{t}=\frac{\varphi \lambda}{\alpha_{i}} \pi_{t}+\frac{\varphi \alpha_{x}}{\alpha_{i}} x_{t}
$$

where at this point we drop the term $i^{*}$ since for brevity we are suppressing all intercepts. As Duffy and Xiao (2007) discuss, this is formally a contemporaneous-data Taylor rule. They show that for calibrated values of structural parameters and policy weights, this leads to a determinate and E-stable equilibrium.

The central bank's observing contemporaneous output and inflation is problematic. We therefore examine the rule

$$
i_{t}=\frac{\varphi \lambda}{\alpha_{i}} \hat{E}_{t-1} \pi_{t}+\frac{\varphi \alpha_{x}}{\alpha_{i}} \hat{E}_{t-1} x_{t}
$$


where the information set for the nowcasts $\pi_{t}^{e}=\hat{E}_{t-1} \pi_{t}, x_{t}^{e}=\hat{E}_{t-1} x_{t}$ is past endogenous variables and exogenous variables. ${ }^{8}$ This again leads to a model of the form (8) with coefficients (9), where $\chi_{\pi}=\varphi \lambda / \alpha_{i}$ and $\chi_{x}=\varphi \alpha_{x} / \alpha_{i}$. We assume that private agents and central banks estimate the same PLM. Since we are here assuming steady-state learning, we also have $\hat{E}_{t-1} \pi_{t+1}=\hat{E}_{t-1} \pi_{t}$ and $\hat{E}_{t-1} x_{t+1}=\hat{E}_{t-1} x_{t}$.

For a sufficiently large $\alpha_{i}$, the model under this Taylor-Type Rule will suffer from indeterminacy. This follows from the Bullard-Mitra result that the determinacy condition is equation (7), from which the critical value of $\alpha_{i}$ can be deduced. The condition for determinacy is

$$
\alpha_{i}<\bar{\alpha}_{i} \equiv \varphi \lambda+(1-\beta) \lambda^{-1} \varphi \alpha_{x} .
$$

If the central bank's desire to stabilize the interest rate is too strong - that is, if condition (13) is not met - then the central bank fails to adjust the interest rate sufficiently to ensure that the generalized Taylor principle (7) is satisfied. To assess this point numerically, we use the calibrated parameter values of Woodford (2003, table 6.1), with $\alpha_{x}=0.048, \varphi=1 / 0.157, \lambda=0.024$, and $\beta=0.99$, which yields approximately $\bar{\alpha}_{i}=0.28$. Woodford's calibrated values of $\alpha_{i}$ are 0.077 or 0.233 (the latter value is from Woodford, 1999). Thus the condition for determinacy does hold for these calibrations.

We next consider stability under learning. For the PLM $\mathbf{y}_{t}=\mathbf{a}+\mathbf{e}_{t}$, we again get the ALM $\mathbf{y}_{t}=\left(\mathbf{M}_{0}+\mathbf{M}_{1}\right) \mathbf{a}+\mathbf{e}_{t}$ and

$$
\mathbf{T} \equiv \mathbf{M}_{0}+\mathbf{M}_{1}=\left(\begin{array}{cc}
1-\alpha_{i}^{-1} \alpha_{x} \varphi^{2} & \varphi-\alpha_{i}^{-1} \lambda \varphi^{2} \\
\lambda-\alpha_{i}^{-1} \lambda \alpha_{x} \varphi^{2} & \beta+\lambda \varphi-\alpha_{i}^{-1} \lambda^{2} \varphi^{2}
\end{array}\right) .
$$

It can be shown that

$$
\operatorname{det}(\mathbf{T})=\beta\left(1-\alpha_{i}^{-1} \alpha_{x} \varphi^{2}\right)
$$

Stability under all values $0<\gamma \leq 1$ requires that

$$
\left|\beta\left(1-\alpha_{i}^{-1} \alpha_{x} \varphi^{2}\right)\right|<1
$$

8. An alternative would be to assume that agents and the policymaker see the contemporaneous value of the exogenous shocks but not the contemporaneous values of $x_{t}$ and $\pi_{t}$. This would not alter our results. 
and it is clear that for given $\beta, \alpha_{x}, \varphi$ this condition will not be satisfied for a sufficiently small $\alpha_{i}>0$. This leads to our next proposition:

Proposition 3. Let $\hat{\alpha}_{i}=\beta(1+\beta)^{-1} \alpha_{x} \varphi^{2}$. For $0<\alpha_{i}<\hat{\alpha}_{i}$, there exists $0<\hat{\gamma}\left(\beta, \varphi, \alpha_{i}, \alpha_{x}\right)<1$ such that the optimal discretionary Taylor-Type Rule (12) renders the rational expectations equilibrium unstable under learning for $\hat{\gamma}<\gamma \leq 1$.

Thus, in addition to the indeterminacy problem for large values of $\alpha_{i}$, the Taylor-type optimal rule suffers from a more serious problem of instability under constant-gain learning for small values of $\alpha_{i}$. The source of this difficulty is the interaction of strong policy responses seen in equation (12) and a large gain parameter. This combination leads to cyclical overshooting of inflation and the output gap. This is particularly evident as $\alpha_{i}$ tends to zero, since in this case, a positive change in inflation expectations $\hat{E}_{t-1} \pi_{t}$ leads to a large increase in $i_{t}$, which in turn leads to large negative changes in $x_{t}$ and $\pi_{t}$ via equations (3) and (4). The severity of this problem depends on the value of $\hat{\gamma}$ in proposition 3. Ideally, stability would hold for all $0<\gamma \leq 1$, but the problem might not be a major concern if $\hat{\gamma}$ is high.

We investigate the magnitude of $\hat{\gamma}$ numerically by computing the eigenvalues of $\gamma \mathbf{T}+(1-\gamma) \mathbf{I}$. As an example, for the Woodford calibration $\beta=0.99, \varphi=1 / 0.157$, and $\lambda=0.024$, we find that with $\alpha_{x}=0.048$ and $\alpha_{i}=0.077$, the critical value $\hat{\gamma} \approx 0.04$. Since estimates in the macroeconomic literature suggest gains in the range 0.02 to 0.06 , this indicates that optimal Taylor-Type Rules may not be stable under learning. ${ }^{9}$ The source of the problem is that with low $\alpha_{i}$ the implied weights on $\hat{E}_{t-1} \pi_{t}$ and especially $\hat{E}_{t-1} x_{t}$ are very high. Under constant-gain learning, this can lead to instability unless the gain parameter is very low. As we demonstrate later, this problem can be avoided by using a suitable expectations-based optimal rule.

We next consider the case in which the exogenous shocks are $\mathrm{AR}(1)$ processes. The literature uses various information assumptions in this setting. Perhaps the most common assumption is that agents see current and lagged exogenous variables and lagged, but not current, endogenous variables. Expectations under this assumption are denoted $\hat{E}_{t} \pi_{t}, \hat{E}_{t} x_{t}, \hat{E}_{t} \pi_{t+1}, \hat{E}_{t} x_{t+1}$. An alternative would be to replace these with $\hat{E}_{t-1} \pi_{t}, \hat{E}_{t-1} x_{t}, \hat{E}_{t-1} \pi_{t+1}, \hat{E}_{t-1} x_{t+1}$, indicating that

9. Milani (2007b) considers a setting in which agents switch between decreasing-gain and constant-gain estimators, depending on recent average mean-square errors. The estimated gains are even higher in the constant-gain regime, at around 0.07 to 0.08 . 
agents only see lagged information. ${ }^{10}$ Whether agents see current or only lagged exogenous shocks is not particularly crucial and does not affect our main results. We therefore follow the most common assumption that expectations are specified as $\hat{E}_{t} \pi_{t}, \hat{E}_{t} x_{t}, \hat{E}_{t} \pi_{t+1}$, and $\hat{E}_{t} x_{t+1} \cdot{ }^{11}$ In contrast, whether agents and policymakers are able to see current endogenous variables is an important issue for stability under learning, as we have already seen. This is why we use the term operationality to indicate an interest rate rule that does not depend on current endogenous variables.

We now assume that the exogenous shocks $g_{t}$ and $u_{t}$ follow $\mathrm{AR}(1)$ processes, that is,

$g_{t}=\mu g_{t-1}+\tilde{g}_{t}$

and

$u_{t}=\rho u_{t-1}+\tilde{u}_{t}$

where $0<|\mu|,|\rho|<1$, and $\tilde{g}_{t} \sim$ i.i.d. $\left(0, \sigma_{g}^{2}\right), \tilde{u}_{t} \sim$ i.i.d. $\left(0, \sigma_{u}^{2}\right)$ are independent white noise processes. We write this in vector form as

$\mathbf{v}_{t}=\mathbf{F} \mathbf{v}_{t}+\tilde{\mathbf{v}}_{t}$.

Under the current assumptions, the PLM of the agents is

$\mathbf{y}_{t}=\mathbf{a}+\mathbf{c v}_{t}$,

and the forecasts are now $\hat{E}_{t} \mathbf{y}_{t}=\mathbf{a}+\mathbf{c v}_{t}$ and $\hat{E}_{t} \mathbf{y}_{t+1}=\mathbf{a}+\mathbf{c F} \mathbf{v}_{t}$. Using the general model (8), the ALM is

$\mathbf{y}_{t}=\left(\mathbf{M}_{0}+\mathbf{M}_{1}\right) \mathbf{a}+\left(\mathbf{M}_{0} \mathbf{c}+\mathbf{M}_{1} \mathbf{c F}+\mathbf{P}\right) \mathbf{v}_{t}$

10. A third alternative, which is occasionally used in the literature, allows agents to see the contemporaneous values of endogenous variables. However, this assumption runs against the requirement of operationality that we want to emphasize here.

11. The standard assumption under rational expectations is that agents have contemporaneous information. Our information assumption takes account of the operationality critique, but nonetheless allows for the possibility of convergence under learning to the rational expectations equilibrium. 
Figure 1. Stability of Optimal Taylor-Type Rule with $\gamma=\mathbf{0 . 0 2}$.

A. Deviation of $x$ from Rational Expectation

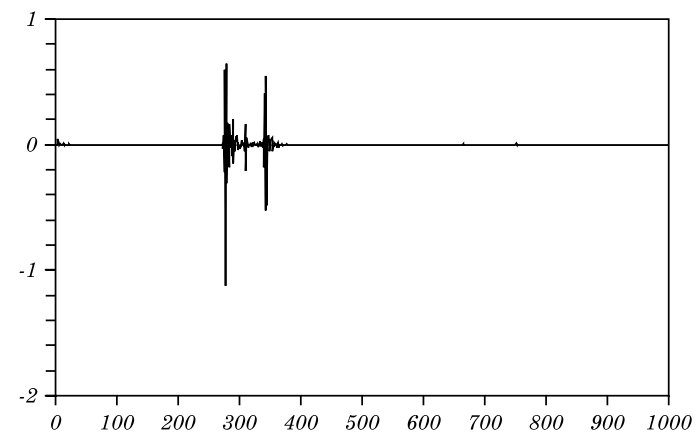

B. Deviation of $\pi$ from Rational Expectation

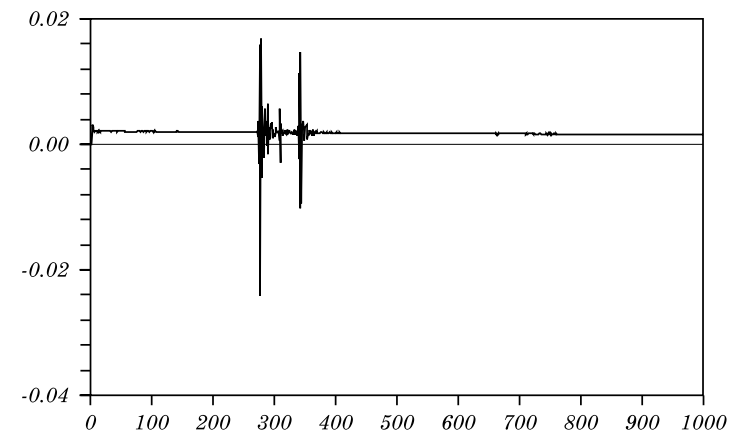

Source: Authors' calculations.

and the E-stability conditions are that all eigenvalues of the matrices $\mathbf{M}_{0}+\mathbf{M}_{1}$ and $\mathbf{I} \otimes \mathbf{M}_{0}+\mathbf{F}^{\prime} \otimes \mathbf{M}_{1}$ have real parts less than one. Here, $\otimes$ denotes the Kronecker product of two matrices. ${ }^{12}$

To examine stability under constant-gain learning, we simulate the model under constant-gain recursive least squares (RLS) estimation of the PLM parameters a and $\mathbf{c} .^{13}$ Under constant-gain RLS, agents discount old data geometrically at the rate $1-\gamma$. Let $\mathbf{a}_{t}, \mathbf{c}_{t}$ denote the estimates based on data through $t-1$. Given these estimates, expectations are formed as $\mathbf{y}_{t}^{e}=\hat{E}_{t} \mathbf{y}_{t}=\mathbf{a}_{t}+\mathbf{c}_{t} \mathbf{v}_{t}$ and

12. In the case of lagged information, the PLM is specified as $\mathbf{y}_{t}=\mathbf{a}+\mathbf{c v}_{t-1}+\eta_{t}$, and the ALM is then $\mathbf{y}_{t}=\left(\mathbf{M}_{0}+\mathbf{M}_{1}\right) \mathbf{a}+\left(\mathbf{M}_{0} \mathbf{c}+\mathbf{M}_{1} \mathbf{c F}+\mathbf{P F}\right) \mathbf{v}_{t-1}+\tilde{\mathbf{v}}_{t}$.

13. See the appendix for the recursive formulation of constant-gain least squares. 
Figure 2. Instability of Optimal Taylor-Type Rule with $\gamma=\mathbf{0 . 0 4}$.

A. Deviation of $x$ from Rational Expectation

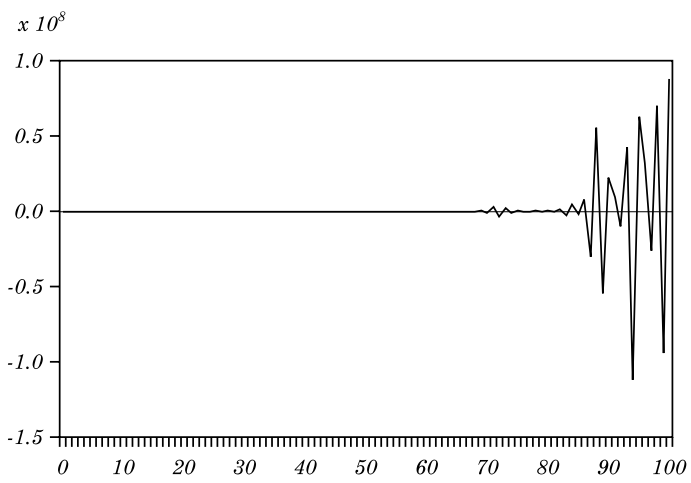

B. Deviation of $\pi$ from Rational Expectation

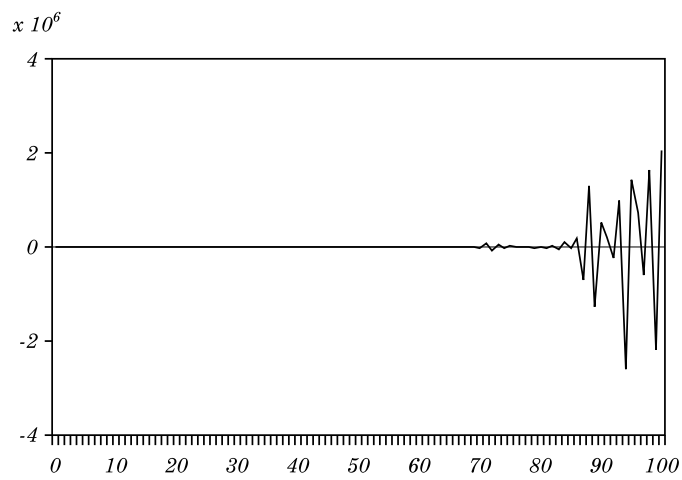

Source: Authors' calculations.

$\mathbf{y}_{t+1}^{e}=\hat{E}_{t} \mathbf{y}_{t+1}=\mathbf{a}_{t}+\mathbf{c}_{t} \mathbf{F} \mathbf{v}_{t}$, and the temporary equilibrium is then given by equation (8) with these expectations.

We use the previous values for the structural parameters and also set $\mu=\rho=0.8$. Simulations of the system indicate instability under constant-gain RLS learning for gain parameters at or in excess of 0.024. Thus, with regressors that include exogenous AR(1) observables, instability arises at even lower gain values than in the case of steadystate learning. Figures 1 and 2 illustrate the evolution of parameters over time under constant-gain RLS learning with the Taylor-Type Rule (12) in stable and unstable cases. ${ }^{14}$

14. In the stable case, the small deviation of $\pi$ from rational expectations, seen in figure 1 , gradually vanishes as the simulation length increases. 


\subsection{Expectations-Based Optimal Rules}

Assume now that at time $t$ the exogenous shocks $g_{t}, u_{t}$ and private-sector expectations $\hat{E}_{t} \pi_{t+1}, \hat{E}_{t} x_{t+1}$ are observed by the central bank. The expectations-based rule is constructed so that it exactly implements equation (11), the first-order condition under discretion, even outside a rational expectations equilibrium for given expectations, as suggested by Evans and Honkapohja (2003b). To obtain the rule, we combine equations (3), (4), and (11) and solve for $i_{t}$ in terms of the exogenous shocks and the expectations. The resulting expectations-based rule is

$$
\begin{aligned}
i_{t}= & \frac{\left(\alpha_{x}+\lambda^{2}\right) \varphi}{\alpha_{i}+\left(\alpha_{x}+\lambda^{2}\right) \varphi^{2}} \hat{E}_{t} x_{t+1}+\frac{\beta \lambda \varphi+\left(\alpha_{x}+\lambda^{2}\right) \varphi^{2}}{\alpha_{i}+\left(\alpha_{x}+\lambda^{2}\right) \varphi^{2}} \hat{E}_{t} \pi_{t+1} \\
& +\frac{\left(\alpha_{x}+\lambda^{2}\right) \varphi}{\alpha_{i}+\left(\alpha_{x}+\lambda^{2}\right) \varphi^{2}} g_{t}+\frac{\lambda \varphi}{\alpha_{i}+\left(\alpha_{x}+\lambda^{2}\right) \varphi^{2}} u_{t} .
\end{aligned}
$$

This leads to a reduced form,

$\mathbf{y}_{t}=\mathbf{M} \hat{E}_{t} \mathbf{y}_{t+1}+\mathbf{P v}_{t}$

Determinacy of the rational expectations equilibrium corresponding to optimal discretionary monetary policy requires that $\mathbf{M}$ has both eigenvalues inside the unit circle. ${ }^{15}$ We again have the condition $\alpha_{i}<\bar{\alpha}_{i}$, where $\bar{\alpha}_{i}$ is given by equation (13).

For stability under learning, first consider the case in which the exogenous shocks $\mathbf{v}_{t}$ are i.i.d. and agents use steady-state learning under constant gain. For this reduced form, the PLM $\mathbf{y}_{t}=\mathbf{a}+\mathbf{e}_{t}$ gives the ALM $\mathbf{y}_{t}=\mathbf{M a}+\mathbf{e}_{t}$ (where $\mathbf{e}_{t}=\mathbf{P} \mathbf{v}_{t}$ ), as discussed in section 1.1. Thus $\mathbf{T}=\mathbf{M}$, and there is a very close connection between determinacy and stability under learning. This leads to proposition 4:

Proposition 4. Assume that $\alpha_{i}<\bar{\alpha}_{i}$ and that the shocks are i.i.d. Then the expectations-based rule, which implements the first-order condition, yields a reduced form that is stable under steady-state learning for all constant-gain rules $0<\gamma \leq 1$.

Provided $\alpha_{i}<\bar{\alpha}_{i}$, so that determinate optimal policy is possible, the expectations-based optimal rule will successfully implement the

15. Equivalently, we need $|\operatorname{tr}(\mathbf{M})|<1+\operatorname{det}(\mathbf{M})$ and $|\operatorname{det}(\mathbf{M})|<1$. 
optimal rational expectations equilibrium: under decreasing-gain learning there will be convergence to the equilibrium, and under small constant-gain learning, it will converge to a stochastic process near the optimal equilibrium. Furthermore, for all constant gains $0<\gamma \leq 1$, there will be convergence to a stationary process centered at the optimal equilibrium.

Second, we examine numerically the case of AR(1) shocks with (constant-gain) RLS learning. For the Woodford calibration $\beta=0.99$, $\varphi=1 / 0.157, \lambda=0.024, \alpha_{x}=0.048$, and $\alpha_{i}=0.077$ (and $\rho=\mu=0.8$ ), we find that learning converges for gain values at or below $\gamma=0.925$. In other words, the expectations-based optimal discretionary rule is quite robustly stable under learning. When the agents have to run genuine regressions, as in the current case, then the IE-stability condition does not imply convergence of constant-gain learning for all $0<\gamma \leq 1$. However, we see that stability does hold even for $\gamma$ quite close to one.

\section{Optimal Policy with Commitment}

For brevity, in the remainder of the paper we assume that $\alpha_{i}=0$, that is, that the central bank does not have an interest rate stabilization objective. ${ }^{16}$ Given the model described in equations (3) and (4) and the loss function (10) with $\alpha_{i}=0$, optimal monetary policy under commitment (from a timeless perspective) is characterized by the condition ${ }^{17}$

$\lambda \pi_{t}=-\alpha_{x}\left(x_{t}-x_{t-1}\right)$,

which is often called the optimal targeting rule. The optimal rational expectations equilibrium of interest has the form

$x_{t}=b_{x} x_{t-1}+c_{x} u_{t}$

and

$\pi_{t}=b_{\pi} x_{t-1}+c_{\pi} u_{t}$

16. See Duffy and Xiao (2007) for an extension to the case in which the central bank also has an interest rate stabilization motive.

17. See, for example, Clarida, Galí, and Gertler (1999) and Woodford (1999). For the exposition, we follow Evans and Honkapohja (2006). 
where we choose the unique $0<b_{x}<1$ that solves the equation $\beta b_{x}{ }^{2}-\left(1+\beta+\lambda^{2} / \alpha_{x}\right) b_{x}+1=0$ and $b_{\pi}=\alpha_{x} / \lambda\left(1-b_{x}\right), c_{x}=-\left[\lambda+\beta b_{\pi}\right.$ $\left.+(1-\beta \rho)\left(\alpha_{x} / \lambda\right)\right]^{-1}$, and $c_{\pi}=-\left(\alpha_{x} / \lambda\right) c_{x}$.

The literature proposes a number of optimal reaction functions that implement the optimal targeting rule (15). Under rational expectations, one obtains the fundamentals-based reaction function

$i_{t}=\psi_{x} x_{t-1}+\psi_{g} g_{t}+\psi_{u} u_{t}$

where

$\psi_{x}=b_{x}\left[\varphi^{-1}\left(b_{x}-1\right)+b_{\pi}\right]$,

$\psi_{g}=\varphi^{-1}$,

and

$\psi_{u}=\left[b_{\pi}+\varphi^{-1}\left(b_{x}+\rho-1\right)\right] c_{x}+c_{\pi} \rho$.

Evans and Honkapohja (2006) show that the reaction function (16) often leads to indeterminacy and always leads to expectational instability. They propose instead the expectations-based reaction function

$i_{t}=\delta_{L} x_{t-1}+\delta_{\pi} \hat{E}_{t} \pi_{t+1}+\delta_{x} \hat{E}_{t} x_{t+1}+\delta_{g} g_{t}+\delta_{u} u_{t}$,

where the coefficients are ${ }^{18}$

$$
\begin{aligned}
& \delta_{L}=\frac{-\alpha_{x}}{\varphi\left(\alpha_{x}+\lambda^{2}\right)}, \delta_{\pi}=1+\frac{\lambda \beta}{\varphi\left(\alpha_{x}+\lambda^{2}\right)}, \delta_{x}=\delta_{g}=\varphi^{-1}, \text { and } \\
& \delta_{u}=\frac{\lambda}{\varphi\left(\alpha_{x}+\lambda^{2}\right)} .
\end{aligned}
$$

Under the interest rate reaction rule (17), the reduced-form model is of the form

$\mathbf{y}_{t}=\mathbf{M}_{1} \hat{E}_{t} \mathbf{y}_{t+1}+\mathbf{N y}_{t-1}+\mathbf{P v}_{t}$

18. In the discretionary case with $\alpha_{i}=0$, the same coefficients would obtain, except that $\delta_{L}=0$. 
with $\mathbf{y}_{t}^{\prime}=\left(x_{t}, \pi_{t}\right)$ and $\mathbf{v}_{t}^{\prime}=\left(g_{t}, u_{t}\right)$. The corresponding rational expectations equilibrium takes the form $\mathbf{y}_{t}=\overline{\mathbf{b}} \mathbf{y}_{t-1}+\overline{\mathbf{c}} \mathbf{v}_{t}$. Evans and Honkapohja (2006) show that the optimal expectations-based reaction function (17) delivers a determinate and E-stable optimal equilibrium for all values of the parameters. It is therefore clearly preferred to the fundamentals-based rule (16).

In connection with constant-gain learning we have the following partial result: ${ }^{19}$

Proposition 5. The expectations-based rule under commitment (17) yields a reduced form for which the eigenvalues of the derivative of the $\mathbf{T}$ map, at the rational expectations equilibrium, are inside the unit circle for all values of the structural parameters.

This result is partial in the sense that the eigenvalues condition is no longer sufficient for stability of constant-gain learning for all $0<\gamma \leq 1$. This is because in the model the regressors include exogenous and lagged endogenous variables.

We now examine numerically the performance of constant-gain RLS learning under the expectations-based optimal rule with commitment. Using Woodford's parameter values (but with $\alpha_{i}=0$ ), we find that constant-gain RLS learning converges for values of the gain parameter below $\hat{\gamma} \approx 0.25$. The inclusion of a lagged variable among the regressors appears to have a significant effect on learning stability for large gains. However, the rule is still robust for all plausible values of the gain parameter.

As noted above, the Duffy and Xiao (2007) formulation under commitment breaks down when $\alpha_{i}=0$ (as it does in the discretionary case). One might investigate numerically the performance of the Duffy-Xiao rule under constant-gain RLS for calibrated values of $\alpha_{i}$. Based on the results in the discretionary case, we are not optimistic about robust learning stability of the Duffy-Xiao rule with commitment.

\section{Alternative Rules for Optimal Policy under Commitment}

This section explores two alternative rules for optimal policy under commitment: the Svensson-Woodford rule and the McCallum-Nelson rule.

19. See the appendix for a proof. 


\subsection{Svensson-Woodford Rule}

Given that the fundamentals-based optimal rules (without interest rate stabilization) lead to problems of indeterminacy and learning instability, Svensson and Woodford (2005) suggest a modification in which the fundamentals-based rule (16) is complemented with a term based on the commitment optimality condition. We again assume that contemporaneous data are not available to the policymaker, so that current values of inflation $\pi_{t}$ and the output gap $x_{t}$ are replaced by their nowcasts $\hat{E}_{t} \pi_{t}$ and $\hat{E}_{t} x_{t}$. This results in the interest rate rule

$$
i_{t}=\psi_{x} x_{t-1}+\psi_{g} g_{t}+\psi_{u} u_{t}+\theta\left[\hat{E}_{t} \pi_{t}+\frac{\alpha_{x}}{\lambda}\left(\hat{E}_{t} x_{t}-x_{t-1}\right)\right]
$$

where $\theta>0$.

The full model is now given by equations (3), (4), and (18). By substituting equation (18) into equation (3), we can reduce this model to a bivariate model of the form

$$
\mathbf{y}_{t}=\mathbf{M}_{0} \hat{E}_{t} \mathbf{y}_{t}+\mathbf{M}_{1} \hat{E}_{t} \mathbf{y}_{t+1}+\mathbf{N y}_{t-1}+\mathbf{P} \mathbf{v}_{t}
$$

where the information set in the forecasts and nowcasts includes current values of the exogenous shocks but not of the endogenous variables. We also assume for convenience that $\mathbf{v}_{t}=\mathbf{F} \mathbf{v}_{t-1}+\tilde{\mathbf{v}}_{t}$ is a known, stationary process. The coefficient matrices are

$$
\begin{aligned}
& \mathbf{M}_{0}=\left(\begin{array}{cc}
-\varphi \alpha_{x} \theta \lambda^{-1} & -\varphi \theta \\
-\varphi \alpha_{x} \theta & -\varphi \theta \lambda
\end{array}\right), \\
& \mathbf{M}_{1}=\left(\begin{array}{cc}
1 & \varphi \\
\lambda & \beta+\lambda \varphi
\end{array}\right), \\
& \mathbf{N}=\left(\begin{array}{cc}
-\varphi \psi_{x}+\varphi \alpha_{x} \theta \lambda^{-1} & 0 \\
-\lambda \varphi \psi_{x}+\varphi \alpha_{x} \theta & 0
\end{array}\right),
\end{aligned}
$$

and

$$
\mathbf{P}=\left(\begin{array}{cc}
0 & -\varphi \psi_{u} \\
0 & 1-\lambda \varphi \psi_{u}
\end{array}\right)
$$


The PLM has the form

$\mathbf{y}_{t}=\mathbf{a}+\mathbf{b y}_{t-1}+\mathbf{c v}_{t}$,

and the $\mathbf{T}$ mapping is

$\mathbf{T}(\mathbf{a}, \mathbf{b}, \mathbf{c})=\left\{\begin{array}{l}{\left[\mathbf{M}_{0}+\mathbf{M}_{1}(\mathbf{I}+\mathbf{b})\right] \mathbf{a}, \mathbf{M}_{1} \mathbf{b}^{2}+\mathbf{M}_{0} \mathbf{b}+\mathbf{N},} \\ \mathbf{M}_{0} \mathbf{c}+\mathbf{M}_{1}(\mathbf{b c}+\mathbf{c F})+\mathbf{P}\end{array}\right\}$.

The usual E-stability conditions are stated in terms of the eigenvalues of the derivative matrices,

$\mathbf{D T}_{\mathbf{a}}=\mathbf{M}_{0}+\mathbf{M}_{1}(\mathbf{I}+\overline{\mathbf{b}})$,

$\mathbf{D} \mathbf{T}_{\mathbf{b}}=\overline{\mathbf{b}}^{\prime} \otimes \mathbf{M}_{1}+\mathbf{I} \otimes \mathbf{M}_{1} \overline{\mathbf{b}}+\mathbf{I} \otimes \mathbf{M}_{0}$,

and

$\mathbf{D T}_{\mathbf{c}}=\mathbf{F}^{\prime} \otimes \mathbf{M}_{1}+\mathbf{I} \otimes \mathbf{M}_{1} \overline{\mathbf{b}}+\mathbf{I} \otimes \mathbf{M}_{0}$,

where $\otimes$ is the Kronecker product and $\overline{\mathbf{b}}$ is the rational expectations value of $\mathbf{b}$.

We compute numerically the E-stability eigenvalues for the Woodford calibration with $\alpha_{x}=0.048$ and $\theta=1.0$. For this case the eigenvalues of DT $\mathbf{D}_{\mathrm{a}}$ are -9.570 and 0.990 , while the eigenvalues of $\mathbf{D T}_{\mathbf{b}}$ are $-10.605,-9.672,0.878$, and -0.0118 . However, $\theta=1.0$ is very close to the lower bound on $\theta$ needed for E-stability (since one root of DT $_{\mathrm{a}}$ is almost one), and the eigenvalues are sensitive to the value of $\theta$. For example, for $\theta=1.5$, the eigenvalues of $\mathbf{D} \mathbf{T}_{\mathbf{a}}$ are -15.975 and 0.949 , while the eigenvalues of $\mathbf{D} \mathbf{T}_{\mathbf{b}}$ are $-17.059,-16.082,0.842$ and -0.011 . Thus, large negative eigenvalues appear. ${ }^{20}$

The calculation of the E-stability eigenvalues suggests that the interest rate rule (18) can be subject to instability if learning is based on constant gain. We now examine numerically the performance of rule (18) under different values of the constant gain using the Woodford calibrated values of the model parameters and $\theta=1.5$. Numerical

20. The eigenvalues of the same model, but with contemporaneous data available, would not deliver large negative eigenvalues in the E-stability calculation for this parameterization. 
simulations show that under the interest rate rule (18), constant-gain RLS learning becomes unstable for values of $\gamma$ at 0.019 or higher.

We also examine numerically the sensitivity of the stability upper bound on $\gamma$ for different values of $\alpha_{x}$, that is, the degree of flexibility of inflation targeting. Table 1 gives the approximate highest value, $\hat{\gamma}$, of the gain for which stability under constant-gain learning obtains. The table shows that robust learning stability of the Svensson-Woodford hybrid rule is very sensitive to the degree of flexibility in inflation targeting. Robust stability obtains only when the central bank is an inflation hawk.

Table 1. Critical Values of $\gamma$ for Stability: Svensson-Woodford Rule

\begin{tabular}{lcccccccc}
\hline$\alpha_{x}$ & 0.01 & 0.02 & 0.03 & 0.04 & 0.05 & 0.06 & 0.08 & 0.10 \\
$\hat{\gamma}$ & 0.185 & 0.060 & 0.035 & 0.020 & 0.018 & 0.014 & 0.009 & 0.007 \\
\hline
\end{tabular}

Source: Authors' calculations.

\subsection{McCallum-Nelson Rule}

McCallum and Nelson (2004) propose a different rule that approximates optimal interest rate policy from a timeless perspective. They suggest that the interest rate be raised above inflation whenever the timeless-perspective optimality condition is above zero. Their rule performs well if $\mathbf{y}_{t}$ is observable, but as McCallum and Nelson (2004) themselves point out, such a rule would be subject to the operationality problem that we have encountered several times: it presupposes that contemporaneous data on inflation and the output gap are available. One way to overcome this problem is to replace unknown contemporaneous data by nowcasts of the variables. In this case, the interest rate rule becomes

$i_{t}=\hat{E}_{t} \pi_{t}+\theta\left[\hat{E}_{t} \pi_{t}+\frac{\alpha_{x}}{\lambda}\left(\hat{E}_{t} x_{t}-x_{t-1}\right)\right]$

Under rational expectations, this rule approximates optimal policy under (timeless-perspective) commitment, provided $\theta>0$ is large.

The model is then given by equations (3), (4), and (20). The model can be reduced to a bivariate model of the form (19), where the 
coefficient matrices are

$$
\begin{aligned}
& \mathbf{M}_{0}=\left(\begin{array}{cc}
-\theta \varphi \alpha_{x} \lambda^{-1} & -\varphi(1+\theta) \\
-\theta \varphi \alpha_{x} & -\varphi \lambda(1+\theta)
\end{array}\right), \\
& \mathbf{M}_{1}=\left(\begin{array}{cc}
1 & \varphi \\
\lambda & \beta+\lambda \varphi
\end{array}\right), \\
& \mathbf{N}=\left(\begin{array}{cc}
-\theta \varphi \alpha_{x} \lambda^{-1} & 0 \\
\theta \varphi \alpha_{x} & 0
\end{array}\right),
\end{aligned}
$$

and

$$
\mathbf{P}=\left(\begin{array}{cc}
1 & 0 \\
\lambda & 1
\end{array}\right)
$$

Using the same parameter values as in the case of the SvenssonWoodford hybrid rule, with $\alpha_{x}=0.048$, we obtain that for $\theta=1.0$, the eigenvalues of $\mathbf{D T}$ are -9.719 and 0.869 , while the eigenvalues of $\mathbf{D} \mathbf{T}_{\mathbf{b}}$ are $-10.780,-9.833,0.750$, and -0.213 . For $\theta=1.5$ the eigenvalues of $\mathbf{D T} \mathbf{T}_{\mathbf{a}}$ are -16.130 and 0.873 , while the eigenvalues of $\mathbf{D T}_{\mathbf{b}}$ are $-17.228,-16.245,0.762$ and -0.172 . The results are very sensitive to $\alpha_{x}$. For $\alpha_{x}=0.100$, we obtain that for $\theta=1.0$ the eigenvalues of $\mathbf{D T} \mathbf{T}_{\mathbf{a}}$ are -22.954 and 0.912 , while the eigenvalues of $\mathbf{D T}_{\mathbf{b}}$ are $-24.042,-23.033,0.835$ and -0.143 . The large negative eigenvalues indicate the potential for instability under constant-gain learning. Using the Woodford calibration (including $\alpha_{x}=0.048$ ) and choosing $\theta=1.5$, we find that constant-gain RLS learning becomes unstable for values of the gain at or above 0.017 .

We again examine numerically the sensitivity of the stability upper bound on $\gamma$ for different values of $\alpha_{x}$, that is, the degree of flexibility of inflation targeting. Table 2 gives the approximate highest value $\hat{\gamma}$ of the gain for which stability under constantgain learning obtains. Comparing the two tables reveals that the stability performance of the McCallum-Nelson rule (20) is about the same as that of the hybrid rule (18) for the same parameter values. Neither rule is robust for many plausible values of the gain parameter. 
Table 2. Critical Values of $\gamma$ for Stability: McCallum-Nelson Rule

\begin{tabular}{lcccccccc}
\hline$\alpha_{x}$ & 0.01 & 0.02 & 0.03 & 0.04 & 0.05 & 0.06 & 0.08 & 0.10 \\
$\hat{\gamma}$ & 0.174 & 0.057 & 0.031 & 0.020 & 0.017 & 0.014 & 0.009 & 0.007 \\
\hline \multicolumn{7}{l}{ Source: Authors' calculations. }
\end{tabular}

McCallum and Nelson (2004) suggest that a preferable alternative to equation (20) is to use forward expectations instead of nowcasts, since this delivers superior results under rational expectations. In this case, the model has no lagged endogenous variables, that is, $\mathbf{N}=\mathbf{0}$ in equation (19). We analyze this case numerically in Evans and Honkapohja (2003a, 2006). Large negative eigenvalues no longer arise in this formulation. However, determinacy and E-stability require a small value of the parameter $\theta$, which can result in significant welfare losses for optimal policy.

\section{Conclusions}

A lot of recent applied research on learning and monetary policy emphasizes discounted (constant-gain) least-squares learning by private agents. We have examined the stability performance of various operational interest rate rules under constant-gain learning for different values of the gain parameter. Since estimates of the gain parameter tend to be in the range of 0.02 to 0.06 for quarterly macroeconomic data, ideally there should be convergence of learning for gain parameters up to 0.1. Based on this criterion, we have found that many proposed interest rate rules are not robustly stable under learning in this sense. An exception to this finding is the class of expectations-based optimal rules in which the interest rate depends on private expectations in an appropriate way. 
APPENDIX

Constant-Gain RLS Algorithm

Suppose the economy is described in terms of a multivariate linear model, which includes possible dependence on lagged endogenous variables. Under least-squares learning, agents have the PLM

$\mathbf{y}_{t}=\mathbf{a}+\mathbf{b y}_{t-1}+\mathbf{c v}_{t}+\mathbf{e}_{t}$

where $\mathbf{a}, \mathbf{b}$, and $\mathbf{c}$ denote parameters to be estimated. Here $\mathbf{y}_{t}$ is a $p \times 1$ vector of endogenous variables. $\mathbf{v}_{t}$ is $k \times 1$ vector of observable exogenous variables, and $\mathbf{e}_{t}$ is a vector of white noise shocks. If the model does not have lagged endogenous variables, then the term by $_{t-1}$ is omitted.

At time $t$ agents compute their forecasts using equation (21) with the estimated values $\left(\mathbf{a}_{t}, \mathbf{b}_{t}, \mathbf{c}_{t}\right)$ based on data up to period $t-1$. Constant-gain RLS takes the form

$\boldsymbol{\xi}_{t}=\boldsymbol{\xi}_{t-1}+\gamma \mathbf{R}_{t}^{-1} \mathbf{Z}_{t-1}\left(\mathbf{y}_{t-1}-\boldsymbol{\xi}_{t-1}^{\prime} \mathbf{Z}_{t-1}\right)^{\prime}$,

$\mathbf{R}_{t}=\mathbf{R}_{t-1}+\gamma\left(\mathbf{Z}_{t-1} \mathbf{Z}_{t-1}^{\prime}-\mathbf{R}_{t-1}\right)$,

where $\boldsymbol{\xi}_{t}^{\prime}=\left(\mathbf{a}_{t}, \mathbf{b}_{t}, \mathbf{c}_{t}\right), \mathbf{Z}_{t}^{\prime}=\left(1, \mathbf{y}_{t-1}^{\prime}, \mathbf{v}_{t}^{\prime}\right)$, and $1>\gamma>0$. The algorithm starts at $t=1$ with a complement of initial conditions. The only difference from standard RLS is that the latter assumes a decreasing gain $\gamma_{t}=1 / t .^{21}$

\section{Proof of proposition 5}

We now sketch a proof of proposition 5 . We examine the formulas given in equations (A7) through (A9) of Evans and Honkapohja (2006, p. 36). Two of the eigenvalues of $\mathbf{D} \mathbf{T}_{\mathbf{b}}$ are 0 , while the remaining eigenvalues are those of the matrix

21. The formal analysis of recursive least squares (RLS) learning in linear multivariate models is developed, for example, in Evans and Honkapohja (1998; 2001, chap. 10). 


$$
\mathbf{K}_{\mathbf{b}}=\left(\begin{array}{cc}
\frac{-\lambda \beta b_{\pi}}{\alpha_{x}+\lambda^{2}} & \frac{-\lambda \beta b_{x}}{\alpha_{x}+\lambda^{2}} \\
\frac{\alpha_{x} \beta b_{\pi}}{\alpha_{x}+\lambda^{2}} & \frac{\alpha_{x} \beta b_{x}}{\alpha_{x}+\lambda^{2}}
\end{array}\right) .
$$

The eigenvalues of $\mathbf{K}_{\mathbf{b}}$ are 0 and $-1<\alpha_{x} \beta\left(2 b_{x}-1\right) /\left(\alpha_{x}+\lambda^{2}\right)<1$. Likewise, two of the eigenvalues of $\mathbf{D} \mathbf{T}_{\mathbf{c}}$ are 0 , while the other two eigenvalues are those of the matrix

$$
\mathbf{K}_{\mathbf{c}}=\left(\begin{array}{cc}
\frac{-\lambda \beta b_{\pi}}{\alpha_{x}+\lambda^{2}} & \frac{-\lambda \beta \rho}{\alpha_{x}+\lambda^{2}} \\
\frac{\alpha_{x} \beta b_{\pi}}{\alpha_{x}+\lambda^{2}} & \frac{\alpha_{x} \beta \rho}{\alpha_{x}+\lambda^{2}}
\end{array}\right) .
$$

The eigenvalues of $\mathbf{K}_{\mathbf{c}}$ are 0 and $\alpha_{x} \beta\left(b_{x}-1+\rho\right) /\left(\alpha_{x}+\lambda^{2}\right)$, which is inside the unit circle unless $\rho$ is negative and large in magnitude. Finally,

$$
\mathbf{D T}_{\mathbf{a}}=\left(\begin{array}{cc}
\frac{-\lambda \beta b_{\pi}}{\alpha_{x}+\lambda^{2}} & \frac{-\lambda \beta}{\alpha_{x}+\lambda^{2}} \\
\frac{\alpha_{x} \beta b_{\pi}}{\alpha_{x}+\lambda^{2}} & \frac{\alpha_{x} \beta}{\alpha_{x}+\lambda^{2}}
\end{array}\right),
$$

and its eigenvalues are 0 and $0<\alpha_{x} \beta b_{x} /\left(\alpha_{x}+\lambda^{2}\right)<1$. 


\section{REFERENCES}

Branch, W.A. and G.W. Evans. 2006. "A Simple Recursive Forecasting Model." Economic Letters 91(2): 158-66.

Bullard, J. 2006. "The Learnability Criterion and Monetary Policy." Federal Reserve Bank of St. Louis Review 88 (May): 203-17.

Bullard, J. and K. Mitra. 2002. "Learning about Monetary Policy Rules." Journal of Monetary Economics 49(6): 1105-29.

Clarida, R., J. Galí and M. Gertler. 1999. "The Science of Monetary

Policy: A New Keynesian Perspective." Journal of Economic Literature 37(4): 1661-707.

Duffy, J. and W. Xiao. 2007. "The Value of Interest Rate Stabilization

Policies When Agents Are Learning." Journal of Money, Credit, and Banking 39(8): 2041-56.

Evans, G.W. and R. Guesnerie. 1993. "Rationalizability, Strong Rationality, and Expectational Stability." Games and Economic Behaviour 5(4): 632-46.

Evans, G.W. and S. Honkapohja. 1998. "Economic Dynamics with Learning: New Stability Results." Review of Economic Studies 65(1): 23-44.

-2001. Learning and Expectations in Macroeconomics. Princeton University Press.

- 2003a. "Adaptive Learning and Monetary Policy Design." Journal of Money, Credit, and Banking 35(6): 1045-72. . 2003b. "Expectations and the Stability Problem for Optimal Monetary Policies." Review of Economic Studies 70(4): 807-24.

- 2006. "Monetary Policy, Expectations, and Commitment." Scandinavian Journal of Economics 108: 15-38.

Evans, G.W., S. Honkapohja and N. Williams. Forthcoming. "Generalized Stochastic Gradient Learning." International Economic Review.

McCallum, B.T. 1999. "Issues in the Design of Monetary Policy Rules." In Handbook of Macroeconomics, vol. 1, edited by J. Taylor and M. Woodford, chap. 23. Amsterdam: Elsevier.

McCallum, B.T. and E. Nelson. 2004. "Timeless Perspective vs. Discretionary Monetary Policy in Forward-Looking Models." Federal Reserve Bank of St.Louis Review 86: 43-56.

Milani, F. 2005. "Adaptive Learning and Inflation Persistence." Working paper 050607. University of California at Irvine, Department of Economics. 
2007a. "Expectations, Learning, and Macroeconomic Persistence." Journal of Monetary Economics 54(7): 2065-82.

— 2007b. "Learning and Time-Varying Macroeconomic Volatility." Working paper 070802. University of California at Irvine, Department of Economics.

Orphanides, A. and J.C. Williams. 2005a. "Imperfect Knowledge, Inflation Expectations, and Monetary Policy." In The InflationTargeting Debate, edited by B. Bernanke and M. Woodford, chap. 5. University of Chicago Press.

. 2005b. "The Decline of Activist Stabilization Policy: Natural

Rate Misperceptions, Learning, and Expectations." Journal of Economic Dynamics and Control 29(11): 1927-50.

Slobodyan, S., A. Bogomolov and D. Kolyuzhnov. 2006. "Stochastic Gradient Learning versus Recursive Least Squares Learning." Working paper 309. CERGE-EI.

Svensson, L.E.O. and M. Woodford. 2005. "Implementing Optimal Policy through Inflation-Forecast Targeting." In The InflationTargeting Debate, edited by B. Bernanke and M. Woodford, pp. 19-83. University of Chicago Press.

Woodford, M. 1999. "Optimal Monetary Policy Inertia." Working paper 7261. Cambridge, Mass.: National Bureau of Economic Research.

- 2003. Interest and Prices: Foundations of a Theory of Monetary Policy. Princeton University Press. 